\title{
Borderline Resectable Pancreatic Cancer: What Have We Learned and Where Do We Go From Here?
}

\author{
Matthew H. G. Katz, MD, Peter W. T. Pisters, MD, Jeffrey E. Lee, MD, and Jason B. Fleming, MD \\ Department of Surgical Oncology, The University of Texas M. D. Anderson Cancer Center, Houston, TX
}

In this issue of the Annals of Surgical Oncology, Stokes et al. make an important contribution to the literature on borderline resectable pancreatic ductal adenocarcinoma (PDAC). ${ }^{1}$ The investigators from the University of Virginia found that $46 \%$ of patients with borderline resectable PDAC (defined using a classification developed at the University of Texas M. D. Anderson Cancer Center [MDACC] over the past decade) completed a multidisciplinary treatment program of neoadjuvant capecitabinebased chemoradiation and surgery and that this group had an overall survival duration that was both favorable (median, 23 months) and similar to that of a historical cohort of patients with potentially resectable PDAC who underwent resection. ${ }^{2,3}$ These data, combined with those from series recently reported by Fox Chase Cancer Center and our own institution, provide strong support for the use of neoadjuvant treatment sequencing in patients with localized PDAC who would be at high risk for marginpositive resection and early treatment failure following a surgery-first strategy. ${ }^{2,4,5}$

Unfortunately, despite the introduction of the concept of borderline resectable disease by the National Comprehensive Cancer Network as early as 2003, these three singleinstitution series-all retrospective studies-represent most of our current knowledge on the topic. Indeed, since the premature closure in 2005 of Eastern Cooperative Oncology Group Trial 1200, the first national trial dedicated to the study of borderline resectable PDAC, no data from prospective trials have been generated to guide the evaluation or management of patients with this stage of disease. ${ }^{6}$ Given this historical context and the recognized

(C) Society of Surgical Oncology 2010

Published Online: 7 December 2010

M. H. G. Katz, MD

e-mail: mhgkatz@mdanderson.org need for improved standardization and quality control in multi-institutional clinical trials for pancreatic cancer, we must ask: How do we incorporate what we have learned thus far into the design of future prospective clinical trials, and what questions need to be answered before we take the critical next step to characterize this challenging group of patients?

\section{WHAT IS THE DEFINITION OF BORDERLINE RESECTABLE PDAC?}

The success of any future trial relies on the adoption of a single objective definition of borderline resectable PDAC. At present, two definitions have been proposed. We developed the MDACC definition, used by Stokes et al., in an attempt to identify patients with primary tumor anatomy, cancer biology, or patient physiology which places them at the limits of compatibility with favorable results after neoadjuvant therapy and surgical resection. ${ }^{3}$ This definition was introduced within the context of a strong institutional preference for the administration of neoadjuvant therapy to all patients with localized PDAC and an opinion that tumor involvement of the superior mesenteric vein and/or portal vein (SMV-PV) (in the absence of arterial involvement) is consistent with potentially resectable anatomy. The criteria for borderline resectable disease were therefore meant neither to justify the role of neoadjuvant therapy among a subset of patients with localized cancers, nor to estimate their potential need for venous resection and reconstruction. ${ }^{8}$

The MDACC anatomic definition differs from that recently proposed by the American Hepato-Pancreato-Biliary Association (AHPBA), Society of Surgical Oncology (SSO), and Society for Surgery of the Alimentary Tract (SSAT), particularly with regards to the classification of tumors with minimal vein involvement. Indeed, the AH$\mathrm{PBA} / \mathrm{SSO} / \mathrm{SSAT}$ definition classifies primary cancers with 
any degree of vein involvement-from slight abutment to outright venous occlusion-as borderline resectable. ${ }^{9}$ Moreover, in contrast to our group, the AHPBA/SSO/ SSAT uses the borderline resectable definition as the basis for recommendations regarding treatment sequencing: surgery is recommended as the standard initial approach for resectable patients, and neoadjuvant therapy (and a heightened awareness of the potential need for SMV-PV resection) is recommended for borderline resectable patients. $^{10}$

We believe that the definition of borderline resectable PDAC proposed by the AHPBA/SSO/SSAT may be somewhat broad. Nonetheless, given national practice patterns that, at present, continue to strongly favor the use of initial surgery among patients with PDAC that does not involve the mesenteric vasculature, and given the survival benefit associated with the use of neoadjuvant therapy for tumors that at least compress the SMV unilaterally, a definition close to that proposed by the AHPBA/SSO/ SSAT should be able to guide management and serve as an inclusion or exclusion criterion for future national trials of treatment for patients with borderline resectable PDAC. ${ }^{4,11}$ Whatever definition is ultimately adopted, it must be objective, clear, and-most importantly-consistently applied.

\section{WHAT TREATMENT ALGORITHM SHOULD INITIALLY BE STUDIED PROSPECTIVELY?}

The data from Stokes et al. supplement available evidence that neoadjuvant treatment sequencing is a rational approach for treating borderline resectable PDAC. ${ }^{1,2,4}$ However, whether preoperative chemotherapy, chemoradiation, or a combination is best is unknown; and the role of additional postoperative therapy, if any, is entirely undefined. At MDACC, the potential oncologic and selective advantages of preoperative therapy are leveraged over a prolonged period of time; in general, we administer systemic chemotherapy first, then consolidating chemoradiation, and finally surgery. Only patients who complete a 4- to 6-month course of nonoperative therapy without disease progression ultimately undergo pancreaticoduodenectomy. Although this strategy has a sound rationale, its benefit over other sequencing patterns (e.g., neoadjuvant chemoradiation followed by chemotherapy or chemotherapy alone) has not been demonstrated. ${ }^{5}$ Provocatively, the data presented by Stokes et al. suggest that equivalent results might be achieved with neoadjuvant chemoradiation alone. ${ }^{1}$ At present, existing data support the study of preoperative chemoradiation-containing regimens in prospective clinical trials.

\section{WHAT CRITERIA SHOULD BE MET TO PROCEED TO LAPAROTOMY?}

Uniform criteria for proceeding to resection upon completion of neoadjuvant therapy must be established. At our institution, and as described by Stokes et al., attempted resection is offered to all patients with borderline resectable PDAC who have no evidence of cancer progression (either local or distant) on presurgical restaging studies. We do not require radiographically evident downstaging to bring patients to the operating room. Such a policy may not be observed at all centers, but its use is rational based on our high rate of $\mathrm{R} 0$ resection among patients with borderline resectable PDAC, particularly when viewed in light of the low rate of radiographic downstaging that is clinically observed.

\section{WHAT SURGICAL STANDARDS ARE NEEDED?}

Although the use of neoadjuvant therapy has been emphasized, the critical importance of a well-performed technical operation for patients with borderline resectable PDAC must not be overlooked. Given the frequent need for segmental resection and reconstruction of the SMV, PV, or hepatic artery to achieve negative resection margins among these patients, their care must be provided by surgeons who are comfortable with techniques of vascular resection and reconstruction at pancreaticoduodenectomy. Moreover, particularly in this group in whom the primary cancer may abut the superior mesenteric artery, meticulous dissection along the periadventitial plane of the vessel at pancreaticoduodenectomy is absolutely essential to maximize the potential for a margin-negative resection. The importance of this technical detail must be reemphasized, given recent evidence that use of a stapling device to remove the surgical specimen may be routine in many centers. ${ }^{7}$ Accurate reporting of such details, preferably using a standard format of documentation, is also critical.

\section{THE NEXT STEP: A MULTI-INSTITUTIONAL TRIAL?}

Because patients with borderline resectable PDAC may be considered to have locally advanced disease by many physicians, they may frequently be denied the opportunity to receive potentially curative therapy. To expand the proportion of patients with localized PDAC who receive such therapy, a thorough understanding of borderline resectable PDAC and its treatment must be rapidly disseminated. In this regard, Stokes et al. have added to the foundation of knowledge on which prospective trials of novel therapeutic strategies for borderline resectable PDAC can-and must-be developed. 
Given the relative infrequency with which patients with this disease stage are seen in any single center, and given the technical demands associated with their surgical care, an initial multi-institutional trial enrolling patients from several experienced treatment centers is urgently needed. The regimen to be tested in such a trial is certainly important; but given the failure of Eastern Cooperative Oncology Group Trial 1200, the most important question to be answered by the trial would be: Is a cooperative study of borderline resectable patients feasible? Robust accrual to and completion of an initial trial would provide a solid backbone on which future studies of therapy for borderline resectable PDAC could be carried out and evaluated. Careful consideration to questions including those posed above, within the context of an established need for a standardized infrastructure of definitions, decision-making processes, and procedures, will help to ensure its success.

CONFLICT OF INTEREST The authors declare no conflict of interest.

\section{REFERENCES}

1. Stokes JB, Nolan NJ, Stelow EB, et al. Preoperative capecitabine and concurrent radiation for borderline resectable pancreatic cancer. Ann Surg Oncol. (in press).

2. Katz MH, Pisters PW, Evans DB, et al. Borderline resectable pancreatic cancer: the importance of this emerging stage of disease. J Am Coll Surg. 2008;206:833-46.
3. Varadhachary GR, Tamm EP, Abbruzzese JL, et al. Borderline resectable pancreatic cancer: definitions, management, and role of preoperative therapy. Ann Surg Oncol. 2006;13:1035-46.

4. Chun YS, Milestone BN, Watson JC, et al. Defining venous involvement in borderline resectable pancreatic cancer. Ann Surg Oncol. 2010;17:2832-8.

5. Brown KM, Siripurapu V, Davidson M, et al. Chemoradiation followed by chemotherapy before resection for borderline pancreatic adenocarcinoma. Am J Surg. 2008;195:318-21.

6. Landry J, Catalano PJ, Staley C, et al. Randomized phase II study of gemcitabine plus radiotherapy versus gemcitabine, 5-fluorouracil, and cisplatin followed by radiotherapy and 5-fluorouracil for patients with locally advanced, potentially resectable pancreatic adenocarcinoma. J Surg Oncol. 2010;101:587-92.

7. Katz MHG, Merchant NB, Brower S, et al. Standardization of surgical and pathologic variables is needed in multicenter trials of adjuvant therapy for pancreatic cancer: results from the ACOSOG Z5031 trial. Ann Surg Oncol. 2010. doi:10.1245/s10434010-1282-y.

8. Evans DB, Erickson BA, Ritch P. Borderline resectable pancreatic cancer: definitions and the importance of multimodality therapy. Ann Surg Oncol 2010;17:2803-5.

9. Callery MP, Chang KJ, Fishman EK, et al. Pretreatment assessment of resectable and borderline resectable pancreatic cancer: expert consensus statement. Ann Surg Oncol. 2009;16:1727-33.

10. Abrams RA, Lowy AM, O'Reilly EM, et al. Combined modality treatment of resectable and borderline resectable pancreas cancer: expert consensus statement. Ann Surg Oncol. 2009;16:1751-6.

11. Simons JP, Ng SC, McDade TP, et al. Progress for resectable pancreatic cancer? A population-based assessment of U.S. practices. Cancer. 2010;116:1681-90. 